\title{
Location based Energy Efficient Scheme for Maximizing Routing Capability of AODV Protocol in MANET
}

\author{
Sudhir Goswami ${ }^{\mathrm{a}}$, Chetan Agrawal ${ }^{\mathrm{b}}$, Anurag Jain ${ }^{\mathrm{c}}$ \\ ${ }^{a, b, c}$ Department of Computer Science \& Engineering \\ ${ }^{a, b, c}$ Radharaman Institute of technology \& Science, Bhopal
}

\begin{abstract}
Nodes in Mobile Ad hoc Network (MANET) are forming a dynamic topology by that the nodes are continuously change their location and also the nodes functioning is dependent on the limited battery capacity that is called energy. The dynamic behavior of network connection is not maintained for long time. All nodes in network are energy dependent and efficient energy utilization is one of the important criteria in MANET. In this paper we proposed a new location based energy efficient scheme with AODV protocol. In this scheme energy dependent nodes are improving the routing capability of AODV protocol on the basis of location based protocol LAR (Location Aided Routing protocol). In network nodes are not aware about their energy status and also recur flooding of routing packets are consumes more energy in network by that the most of the energy is wasted in connection establishment. LAR protocol reduces that possibility of destination finding by maintain the record of location of each node in network respect to other nodes. It implies that every node in network is maintaining the record of other nodes (participating in routing procedure) with their current location and speed. If the nodes in network are know about the energy status and also about the status of location of receiver that reduces the energy consumption. The main aim of proposed scheme is to enhance the energy utilization in network. The performance of normal AODV, AODV with LAR and AODV with LAR and Energy is illustrate here and observe that the LAR protocol reduces the energy consumption and prolog the network lifetime that completely depend on the energy of mobile nodes.
\end{abstract}

Index Terms: AODV, LAR, Energy, Routing, MANET.

(C) 2015 Published by MECS Publisher. Selection and/or peer review under responsibility of the Research Association of Modern Education and Computer Science

\section{Introduction}

A mobile ad-hoc network [1] is a group of number of devices that can move and communicate with each other without any fixed infrastructure or centralized administration. With respect to the flexibility of mobility, the mobile ad-hoc network can be installed in minimum time at low cost. It is not dependent on current

* Corresponding author. Tel.:

E-mail address: 10188sudhir@gmail.com, chetan.agrawal12@gmail.com 
infrastructure of network. The mobile node can move while communicating, the base stations are fixed and as the node goes out of the range of a base station, it gets into the range of another base station. These nodes generally have a limited range of transmission. Here each node looks forward to its neighbor nodes in order to forward the packets. As we know that the nodes of mobile Ad-hoc network can also perform as a routers and end users. So it is possible that the nodes can also forward the data between different nodes of a network. By nature these types of networks are suitable for situations where either no fixed infrastructure exists or deploying network is not possible. Adhoc mobile networks have found many applications in various fields like military, emergency, conferencing and sensor networks. Each of these application areas has their specific requirements for routing protocols.

Ad hoc network will typically have a dynamic topology, since the network nodes are mobile, which will have profound effects on network characteristics. Network nodes will often be battery powered, which limits the capacity of CPU, memory, and bandwidth. The nodes in a network are battery dependent for their communication. So the most important parameter for optimization is energy conservation. The energy [2] is required to nodes in network for communicating with each other and for every data sending and receiving the nodes are consumes the energy. It means that the each functioning of nodes in network is depending on the energy. In MANET the nodes energy source is not replaced and charged at same instant that the energy depleted or almost discharge in network, by that the energy is the important constraint.

Routing [2,3] is the most important function as a basic data transfer scheme between wireless nodes. The routing is based on the implicit trust relationship between the participating devices. Responsibilities routing Home exchange routing information, and find possible path between the source and destination based on various scales, and maintenance of the road

The major requirements [1] of a routing protocol are

(1) Minimum route attainment delay,

(2) At the time of path break reestablishment of route Quickly

(3) Loop-free routing

(4) Distributed routing protocol,

(5) Low control overhead

(6) Scalability with network size

The challenges are [4] resulting from the unique properties of MANET. Network topology node mobility, which may call for the package lost affect, uninterrupted path, the division of the network and the difficulty in allocating resources. Generally limited resources of wireless contract regarding the battery, memory and calculating power supply

The main drawback in MANET is to forming the dynamic connection between sender and receiver and also the nodes in network are not attentive about the location information of mobile nodes. If the nodes are known the location then the flooding of route request and route reply are minimizes in network because the senders are only flood the routing packets in the direction were the destination are located. It means the problem of most of the energy consumed in connection establishment the major issue that will we solved in this research through do location based routing scheme. Routing protocol design should consider these factors based on the scheme of routing information update, routing protocols in wireless ad- hoc networks known as active protocols or table driven protocols second is reactive protocols or on-demand protocols and third one is hybrid protocols. The purposed scheme presented in related articles reveal particular routing requirements which can be successfully fulfilled by a number of protocols. However, the differences in these routing approaches make some protocols more recommendable than others. One must be able to choose the most suitable option from a vast number of geographic routing possibilities, but this poses a lot of difficulties. Therefore, this examination comes as an assist to those interested in location based routing.

This paper has organized into nine major section including introduction. Second section is for AODV routing protocol. Third section shows the location based routing protocol. Fourth section gives an idea of previous 
approached used in this area. In section five the problem has been discussed. The section six shows the proposed methodology and algorithms. Section seven provides the detail of simulation environment and result analysis. Finally the paper has concluded in section eight. The references are shown in section nine.

\section{Ad-hoc On demand Distance Vector or AODV Protocol for Routing}

AODV [5] the same processes as DSR key apply. Discover the path to a destination using the route request (RREQ) and route reply (RREP) sequence, performs maintenance for the failure of the link road through the publication of route error message (RERR) to the source. AODV is trying to improve the DSR to maintain routing tables in the contract, so that data packets do not contain the source path to destination. Each node maintains a route for each item on the agenda of interest, including the following fields: on one hand, and the next hip, of hops number, destination sequence number and expiration time. When a source node station, who asked how to find the way to a destination, and intermediate nodes, the reverse path forwarding message creation, pointing to the node receiving the request. Thus RREP travels the contrary create routes when forwarded RREQ, without incurring the full path to the head. When RREP travels along the return path, each node makes direct links to be used later to route data packets between the source and destination. When the RREQ source node sends a sequence number to the top of the destination is assigned. It allows intermediate nodes to respond only if recently know RREP path to the destination (with the same or greater sequence number). It is removed and the opposite paths to transmit routing tables if not used within a certain period of time. AODV advantages can be summarized as follows:

(1) Route never have and carried in the header of a packet.

(2) Nodes always contention the table of routing

(3) AODV uses a scheme of destination sequence number in order to bound the chances of an intermediate node replying with stale information to a RREQ packet.

\section{Location Based Protocol Description}

Location Aided Routing (LAR) [6, 7]: Ko and Vaidya present the LAR protocol which utilizes location information to minimize the search space for route discovery towards the destination node. LAR aims to reduce the routing overhead for the route discovery and minimizes the energy consumption. This protocol proposes two specific schemes to control the flooding and for more flooding in network lot of energy is wasted for connection establishment. The one is expected zone and requested zone. Expected zone shapes a circle considering the movement of destination node. Requested zone shapes a rectangular which includes source node and expected zone. In each zone, flooding is restricted differently. And another one considers the distance to destination node. If distance is longer than the previous node, then RREQ packets are dropped. In the result, LAR is powerful. But the basic assumption must cause big overheads, since all nodes must know the location information of all nodes in the network. Consequently it is need to reduce these overheads to optimize the location aware protocol. The energy is limited resource of communication in MANET and LAR is reduces the flooding and enhances the routing capability of AODV routing protocol.

\section{Previous Work Accomplished}

In this research [8] paper is presenting an Energy-Efficient Routing protocol that will improve the utilization of link by balancing the energy consumption between utilized and underutilized nodes to meet the above challenge. The protocol deals with various parameters as Residual Energy, Bandwidth, Load and Hop Count for route discovery. The failure of any node in the route when the transmission of data packet is in progress leads to the degradation of the QoS (Quality of Service). To overcome with this issue, the paper proposes two methods for maintenance of the route. 
In this paper [9] Energy Efficient Location Aided Routing Protocol (EELAR) is proposed that is an optimization to the Location Aided Routing (LAR). EELBR makes significant reduction in the energy consumption of the mobile nodes batteries through limiting the area of discovering a new route to a smaller zone. Thus, control packets overhead are significantly reduced and the mobile nodes life time is increased. The simulation results show that there is a tradeoff between decreasing control overhead by increasing number of areas and increasing route loss by increasing the number of network areas due to node mobility.

This recent research [10] proposes the Reliable Minimum Energy Cost Routing (RMECR). RMECR can increase the operational lifetime of the network using energy-efficient and reliable routes. In the design of RMECR, we used a detailed energy consumption model for packet transferrin wireless ad hoc networks. RMECR is designed for two types of $\mathrm{N} / \mathrm{W}$ : one is the hip-hop relaying ensure reliability and ending to end retransmission ensure reliability. General approach has been used, which is used in the RMECR also designed to develop an algorithm of art energy efficiency directive state for ad hoc wireless networks, ie the minimum energy reliable guidance (RMER). RMER find ways to reduce energy consumption to pass the package. Proposes RMECR networks with the hip-hop relay reliability and provide the link layer and network with end to end resend operations provide end-to-end reliability.

In this paper [11] the author has proposed a comparison between two different protocols in the context of energy conservation and orientation of public spending. The proposed work is divided into two units called Energy methodology Node Aware, which includes the cost of police in Maine Max guidance algorithm battery (CMM-BCR) and the destination drive, including the effect of the algorithm for site estimate based mobility (DREAM) routing Protocol diameter. These two are applied together Over on routing protocol demand, which is a dynamic unit source routing protocol (DSR), and the sequence of destination distance vector routing protocol (DSDV) routing tables based on the protocol. General simulation shows that when there is use of energy scheme with DSR it seems to enhanced results with respect to DSDV. So that the energy efficient methodology is a primary goal in ad-hoc networks so the objective is to identify the batter protocol between two if the protocols with respect to energy and enhance the lifetime of the network.

\section{Problem Identification}

The routing in MANET is completely dependent on the flooding of routing packets in network by that the nodes energy is wasted in flooding. Due to limited working capacity of mobile nodes is the major problem in MANET. In MANET the energy is the limited resource because there is a no source is available in network by that the nodes are regain their energy. It means if the node is lost their energy then the battery replacement is only the option to retain the node in network with full capability and the second one option is to utilize the energy of nodes efficiently. The meaning of efficient use of battery power is to reduce the possibility of packet loss and retransmission in network. The routing protocol has no capability to reduce the motion of mobile nodes that is the major region of link breakage and energy wastage. The energy efficient routing scheme is utilizes the nodes power in communication.

\section{Proposed Scheme for Improving Energy Efficiency}

Here we define for energy as well as location base routing, here very first we create mobile node and then set sender and receiver node and all node set initial energy as a random after that, sender broadcast routing packet to find receiver, in route broadcasting time energy module base we find out intermediate node energy if energy value is greater than threshold value then we insert that node in route else eliminate that route path, after that we find out receiver node on the bases of threshold energy base routing and receivers sends acknowledgment packet to the actual sender, but after some time any node move and connection break down than receiver uses LAR (Location aided routing) and sends location information to sender node time to time, that message help full for finding receiver from minimum overhead bases because LAR uses direction base routing and increases 
the performance of the network. Our objective is to get reliable path by which cost of energy can be reduced. To this end, reliability and cost of energy of path should be careful in path selection.

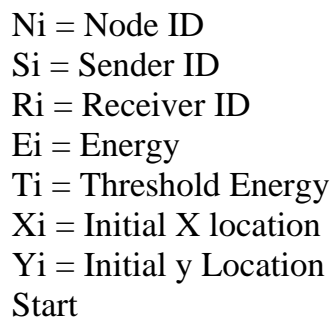

Node Si uses AODV Broadcast Route Request

\{

If (Node exist in Range \&\& Node Energy $\geq 10$ ) // 10 Joule threshold value

\{Received Route Packet

Match Ri ID to Self ID

If (Not Match)

$\{$ Initiate Ei of Node into Packet Header

Forward Packet to Next Hop

If(Ri ID $==$ Self Current Node ID)

\{Receiver Found

Check all Available Path from Si to Ri

Select Maximum Energy Path

\}

Else

\{Node not Found

\}

\section{Location Identification Module}

Routine: Location Information

Si Route break Detected

Ri Calculate Location with Respect to $\mathrm{Si}$

Periodically Send XRi, YRi and \& Speed into Si Node // X and Y location

Set Si Receive location information from Ri

Si Calculate XRi and YRi Coordinate

\{

If (Ri Displacement DXRi, DYRi\&\&Ri not Found) //Displacement from X and Y position

$\{$ NewXRi $=$ Old XRi + DXRi;

New YRi = Old YRi + DYRi;

$\mathrm{DX} \mathrm{Ri}=\mathrm{X}_{2}-\mathrm{X}_{1}$

$\mathrm{DYRi}=\mathrm{Y}_{2}-\mathrm{Y}_{1}$

Distance Cost $\sqrt{D X R i^{2}+D Y R i^{2}} / /$ Estimated cost of receiver

\}

Si Search Ri using Route Request Information and Distance Cost of Ri

Exit

\} 


\section{Simulation Environment and Evaluated Results}

The simulations of proposed work has been performed on the network simulator 2.31 [11] and provides a simulation of a dedicated mobile networks. The simulation parameters has been shown in Table 1. Movement model implement random waypoint for simulation, which is a node starts at a random position, the timeout and pause, and then moves to another position randomly choosing speed $35 \mathrm{~m} / \mathrm{s} .512$ bytes of packet size and baud rate of 4 packets / sec.

\subsection{Simulation Parameters}

The following are the simulation parameters are considered for simulating the LAR protocol with Energy.

Table 1. Simulation Parameters for Case Study

\begin{tabular}{|l|l|}
\hline Examined Protocol & AODV \\
\hline Number of nodes Used & 50 \\
\hline Protocol of Location & LAR \\
\hline Simulation area Dimension & $800 \times 600$ \\
\hline Total time of Simulation & $100 \mathrm{~ms}$ \\
\hline Range of Node & $250 \mathrm{~m}$ \\
\hline Type of Traffic & CBR, 3pkts/s \\
\hline Size of Packet & 512 bytes \\
\hline Traffic Connections & TCP/UDP \\
\hline Maximum Speed $(\mathrm{m} / \mathrm{s})$ & 35 \\
\hline Node movement & random \\
\hline
\end{tabular}

\section{Performance Metrics}

In this simulation there is a use of several performance metrics in order to measure up to the proposed protocol of AODV with the existing one [12]. The following metrics were considered for the comparison were

Throughput: It is calculation of No of packets received by the network in a unit amount of time.

Packet delivery fraction (PDF): The ratio between the No of packets sends by sending node to the No of packets actually received by the end nodes.

End to End delay: Delay at the end is calculated by the average data packet latency.

Routing load: The No of packets of routing transmitted for each single data packet send to the destination.

This section presents the result that illustrates the performance of proposed AODV with LAR and Energy. The performance of AODV with LAR is almost same in some matrices.

\subsection{Normal Routing Load analysis}

The number of routing packets is delivered in network for established connection in between sender and receiver. The routing load is measured through the number of routing packets and number of received at destination in a given simulation time. The graph in figure 1 shows the performances of three protocols are observed i.e. only AODV routing protocol, AODV with LAR and AODV with Energy and LAR. The Location Aware Routing (LAR) protocol is useful for maintaining the location information of mobile nodes in dynamic 
environment. The LAR protocol is improves the routing mechanism of AODV protocol by that the flooding of routing packets is minimized as compare to normal AODV routing. Here in case of AODV about 3200 routing packets are received in network for connection establishment. In case LAR with AODV protocol about 2000 routing packets is received in network but in case of AODV with Energy and LAR about only 1500 routing packets are received in network. It implies that in case of adding energy factor the LAR provides the better performance in a given simulation time and utilizes the energy for communication in network.

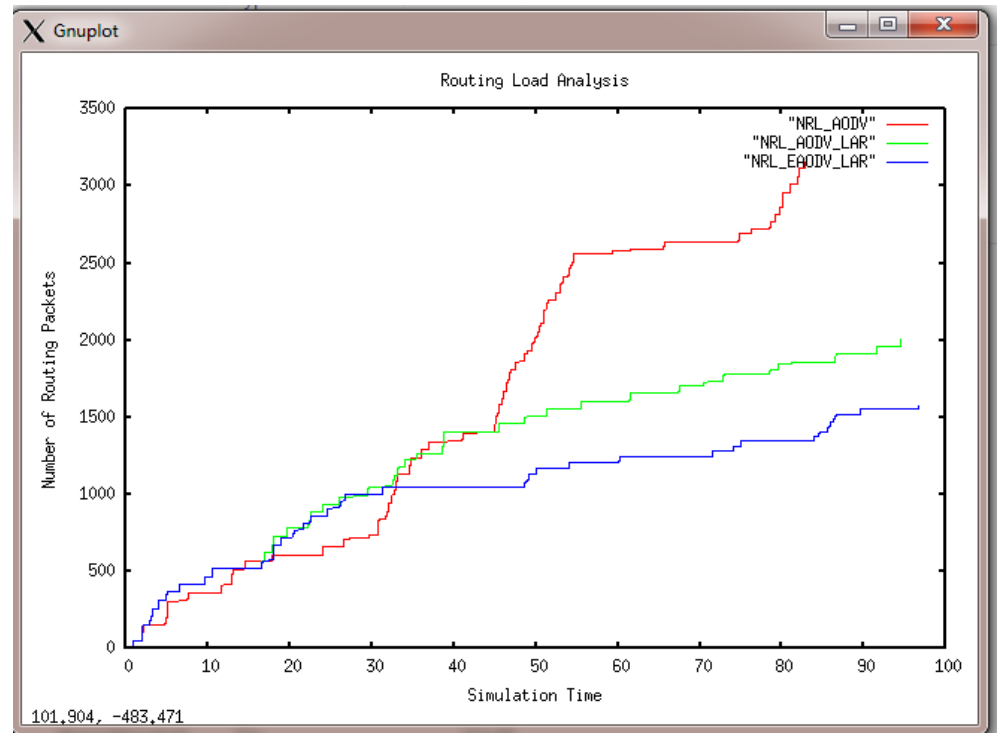

Fig 1. NRL Analysis

\subsection{Throughput Analysis}

Throughput is one of the important matrices to measure the network performance per unit of time in network. In figure 2 the graph shows the performance of these three protocols is observed i.e. AODV, AODV with LAR and AODV with LAR and Energy. The energy factor in first two protocols are not included, means the network life time is not limited and also not evaluated the network life time of these two protocols. The energy factor in third one is included and it also provides the equal throughput performance. The throughput in case of proposed AODV with proposed LAR and Energy is slightly down at the end of simulation that signified the depletion of nodes energy and continues the routing procedure up to end of simulation.

\subsection{Packet Delivery Ratio (PDR) Analysis}

The percentage of data is successfully received in network is evaluated through this graph in case of AODV, AODV with LAT and AODV with LAR and Energy. The percentage performance of AODV protocol is less as compare to rest of both protocols, which is about $89 \%$ at the end of simulation. The PDF performance of LAR and proposed LAR with Energy is almost equal and provides the PDF performance about $97 \%$ at the end of simulation. Figure 3 The LAR protocol is improves the packet receiving percentage ratio and also able to maintained the strong connectivity between the links in dynamic network. The energy loss due to link breakage is about negligible because the performance is equal to LAR. The link breakage and possibility of retransmission is clearly visualized in pure AODV routing and through LAR it is possible to remove. 


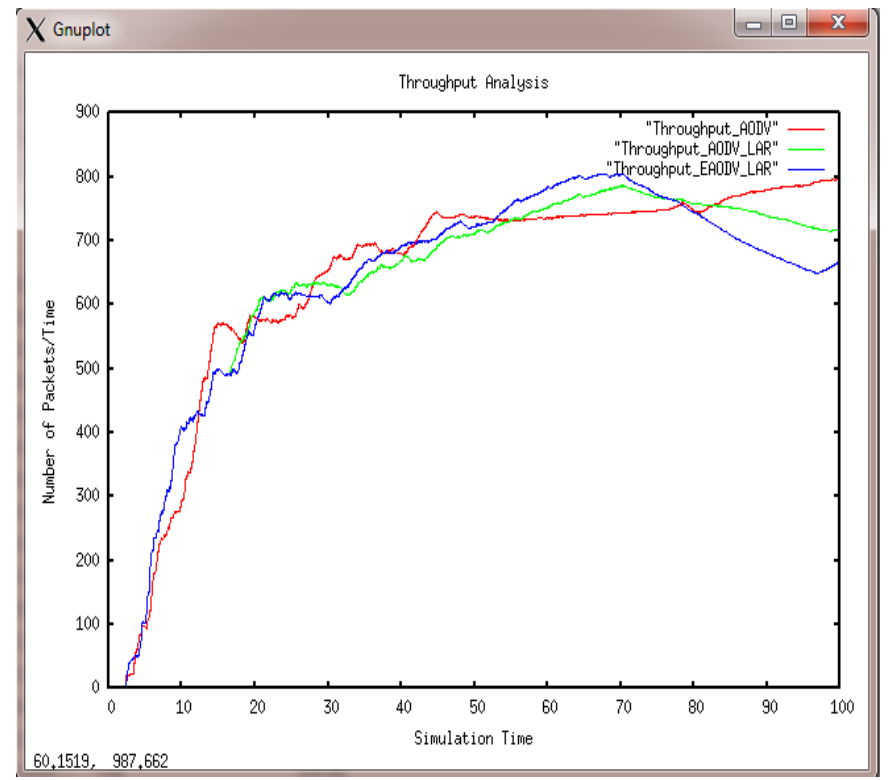

Fig 2. Throughput Analysis

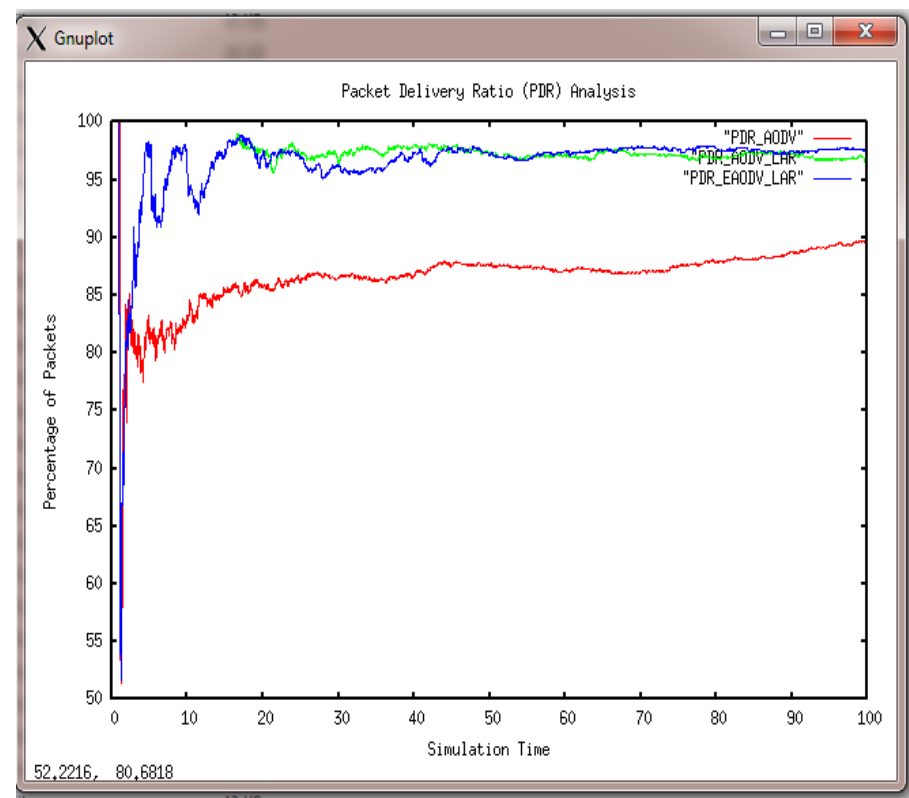

Fig 3. PDR Analysis

\section{Other Comparative Study}

Here the simulation has done with different nodes. Here the comparative results are shown. Here the parameter packet deliver ratio, routing load and throughput has shown. The graphs shows the comparison with three protocols those are AODV, Location aided Routing and Energy efficient Location Aided Routing. the 
conclusion of graph shows the EAODV shows the batter result in packet delivery ratio and routing load. Here the throughput should be enhancing.

Table 2. Other Simulation Parameters for Case Study

\begin{tabular}{|l|l|}
\hline Examined Protocol & AODV \\
\hline Number of nodes Used & $20,40,60,80,100$ \\
\hline Protocol of Location & LAR \\
\hline Simulation area Dimension & $800 \times 600$ \\
\hline Total time of Simulation & $100 \mathrm{~ms}$ \\
\hline Range of Node & $250 \mathrm{~m}$ \\
\hline Type of Traffic & CBR, 3pkts/s \\
\hline Size of Packet & 512 bytes \\
\hline Traffic Connections & TCP/UDP \\
\hline Maximum Speed $(\mathrm{m} / \mathrm{s})$ & 35 \\
\hline Node movement & random \\
\hline
\end{tabular}

The table shows the result of packet delivery ratio. With respect to all three packet at time $99.99 \mathrm{~ms}$

Table 3. PDR Result

\begin{tabular}{|l|l|l|l|}
\hline & AODV & LAR & ELAR \\
\hline 20 & 93.92 & 96.05 & 93.22 \\
\hline 40 & 94.85 & 97.14 & 96.95 \\
\hline 60 & 95.54 & 94.3 & 96.79 \\
\hline 80 & 93.55 & 93.69 & 95.51 \\
\hline 100 & 91.84 & 91.32 & 94.06 \\
\hline
\end{tabular}

\section{Packet Delivery Ratio}

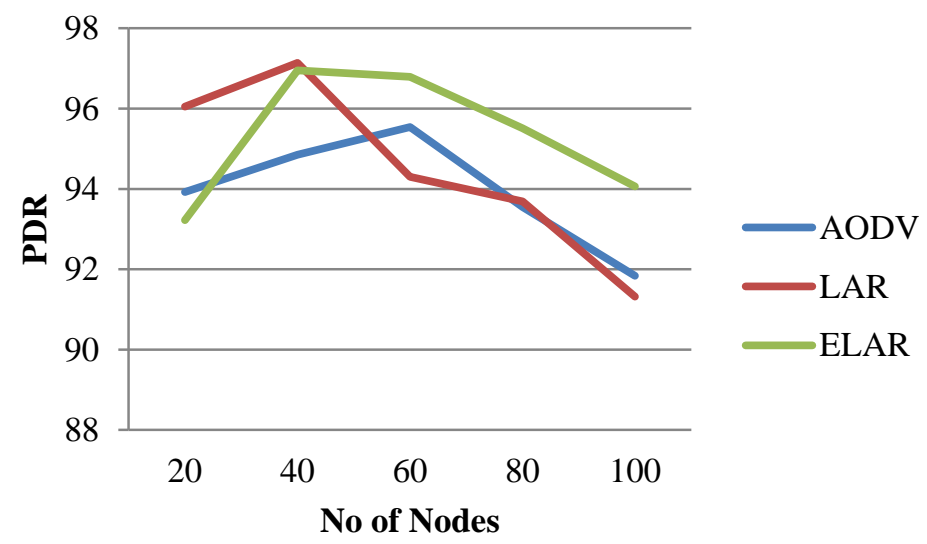

Fig 4. Comparative Graph for PDR 
Here the detail of routing load has been presented. The graph shows the conclusion that when the network is large the routing load will increase.

Table 4. Routing Load Result

\begin{tabular}{|l|l|l|l|}
\hline & AODV & LAR & ELAR \\
\hline 20 & 235 & 202 & 1060 \\
\hline 40 & 1754 & 1605 & 1476 \\
\hline 60 & 2801 & 3855 & 2339 \\
\hline 80 & 4519 & 4933 & 3195 \\
\hline 100 & 5202 & 7165 & 7101 \\
\hline
\end{tabular}

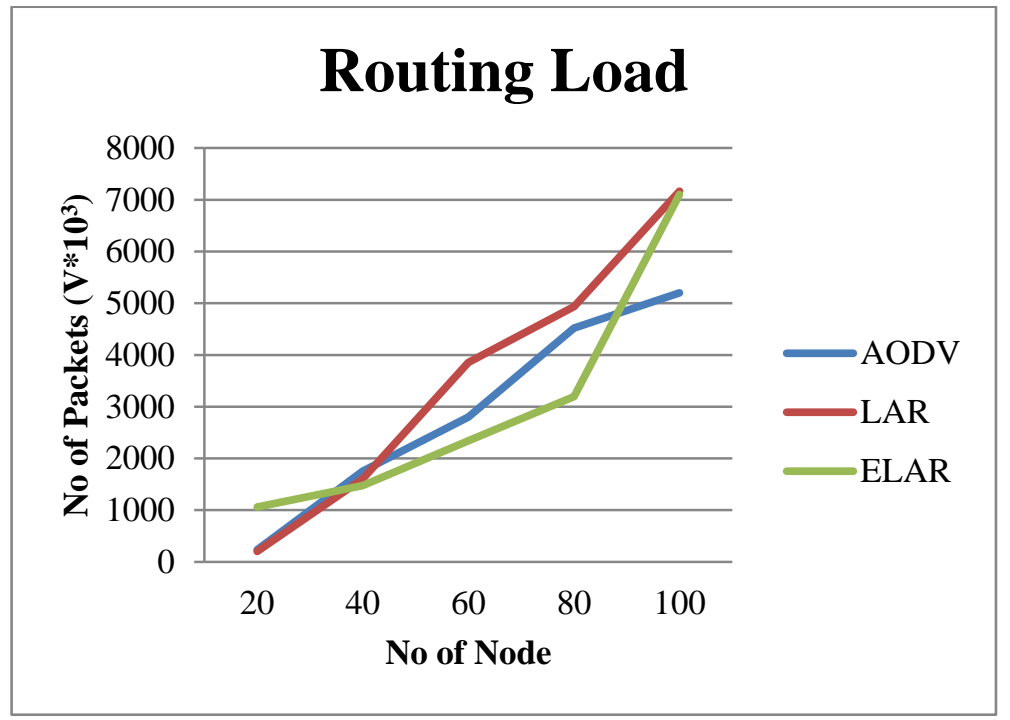

Fig 5. Comparative Graph for Routing Load

The throughput of all method has compared here in which it seems to be that the Energy LAR gives the batter results with respect to LAR but here the AODV have the more efficient in throughput

Table 5. Throughput Result

\begin{tabular}{|l|l|l|l|}
\hline & AODV & LAR & ELAR \\
\hline 20 & 828 & 521.081 & 521.81 \\
\hline 40 & 934 & 691.975 & 787.813 \\
\hline 60 & 772.4 & 586.78 & 597.721 \\
\hline 80 & 982.62 & 582.417 & 551.902 \\
\hline 100 & 988.11 & 540.1 & 683.23 \\
\hline
\end{tabular}




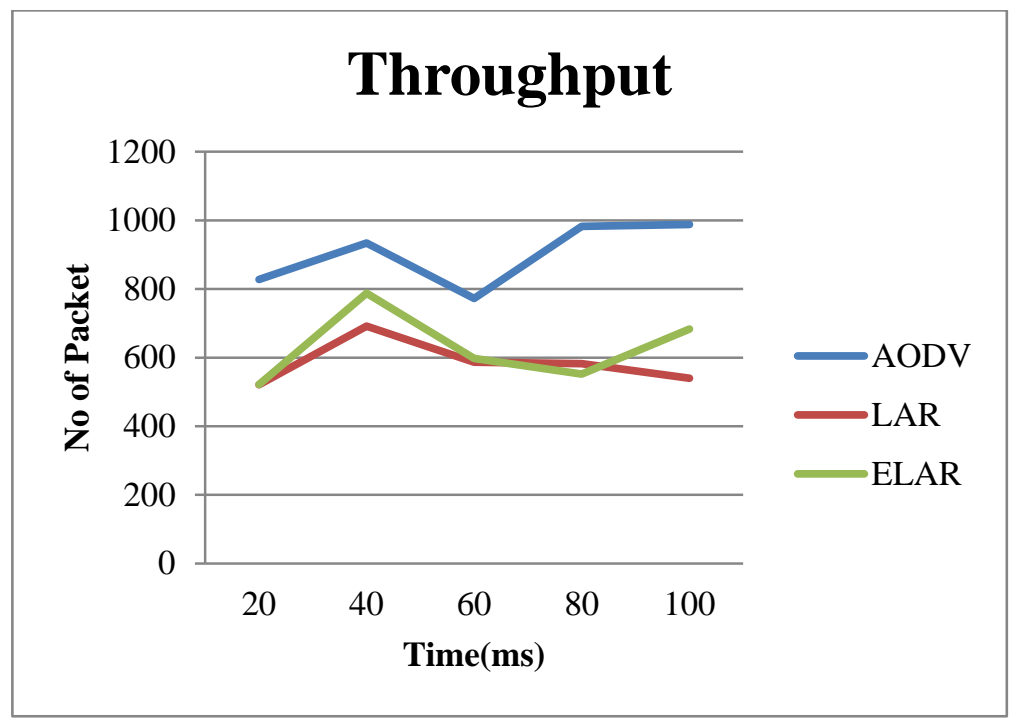

Fig 6. Comparative Graph for Throughput

\section{Conclusion \& Future Work}

The number of nodes in MANET considered dependent on batteries for their energy. So the most important parameter for optimization is energy conservation. It has been further concluded that due to the dynamically changing topology and infrastructure less, decentralized characteristics, location information and power awareness is hard to achieve in MANET. Hence, location awareness and power awareness mechanisms should be built-in features for all sorts of applications based on ad hoc network. In this paper the LAR has fulfill the scarcity of location unawareness by that the power consumption or energy consumption of nodes are more utilizes in routing packets instead of retransmission of data due to link breakage. The proposed AODV with LAR and Energy approach is reduces the flooding of routing packets and provides the performance as equal to AODV with LAR. The proposed approach is improving the capability of AODV routing protocol and prolog the network life time.

In future we focus on LTE (Long Term Evolution) Technology and effort will be made to propose a solution for routing in Ad Hoc networks by tackling next core issues of secure and power aware/energy efficient routing.

\section{References}

[1] C. S. R. Murthy, B. S. Manoj, “Ad-Hoc Wireless Networks: Architectures and Protocols”, Pntice Hal, 2004.

[2] Natalia Vassileva, Francisco Barcelo-Arroyo, "A Survey of Routing Protocols for Maximizing the Lifetime of Ad Hoc Wireless Networks", International Journal of Software Engineering and Its Applications, Vol. 2, No. 3, pp. 77-90, July, 2008.

[3] Sunil Taneja and Ashwani Kush, "A Survey of Routing Protocols in Mobile Ad-Hoc Networks" IJIMT, Volume. 1, No. 3, Aug 2010.

[4] Sudhir Goswami, Chetan Agrawal and Anurag Jain, "A Study of Energy Efficiency Location based Routing Protocols in MANET", International Journal of Scientific \& Engineering Research (IJSER), Vol 5, Issue 6, June 2014. 
[5] C. E. Perkins, E. M. Royer, "Ad-Hoc On-Demand Distance Vector Routing", Workshop on Mobile Computing Systems and Applications , pp. 90-100, IEEE Feb 1999.

[6] Y. Ko, N. Vaidya, Location-Aided Routing (LAR) in mobile ad hoc networks, in: Proceedings of ACM MobiCom, October 1998, pp. 66-75.

[7] Y.-B. Ko, N.H. Vaidya, Geo TORA, “A protocol for geocasting in mobile ad hoc networks”, pp.240249 IEEE ICNP, Nov 2000.

[8] Supriya Srivastava, A.K. Daniel, R. Singh and J.P. Saini, "Energy-Efficient Position Based Routing Protocol for Mobile Ad-Hoc Networks" International Conference on Radar, Communication and Computing (ICRCC), pp. 18-23 Dec 2012.

[9] H.Vijayakumar, M.Ravichandran, "Efficient Location Management of Mobile Node in Wireless Mobile Ad-hoc Network", National Conference on Innovations in Emerging Technology, pp.77-84, India.17 \& 18 IEEE Feb, 2011.

[10] Javad Vazifehdan, R. Venkatesha Prasad, Ignas Niemegeers, "Energy Efficient Reliable Routing Considering Residual Energy in Wireless Ad-Hoc Networks", pp. 1-14, IEEE 2013.

[11] Sheetal Sisodia, Sandeep Raghwanshi, "Performance Evaluation of a Table Driven and On-Demand Routing Protocol in Energy Constraint MANETs" IEEE Conference on Computer Communication and Informatics (ICCCI -2013), pp. Jan. 04 - 06, IEEE 2013.

[12] "Introduction of network simulator 2" accessed from http://www.isi.edu/nsnam/ns/index.html, accessed on $10 / 01 / 2015$.

[13] Y.-C. Tseng, S.-Y. Ni, and E.-Y. Shih, "Adaptive approaches to relieving broadcast storms in a wireless multihop ad hoc networks," IEEE Transaction on Computers, vol. 52, pp. 545- 557, 2003.

[14] J. Cartigny and D. Simplot, "Border node retransmission based probabilistic broadcast protocols in ad hoc networks," Telecommunication Systems, vol. 22, pp. 189-204, 2003.

[15] C Chen, Chin-Kai Hsu and Hsien-Kang Wang, "A distance-aware counter-based broadcast scheme for wireless ad hoc networks", Military comm. Conference- 2005, MILCOM 2005 IEEE 17-20 Oct 2005 pages 1052-1058 Vol-2.

\section{Author(s) Profiles}

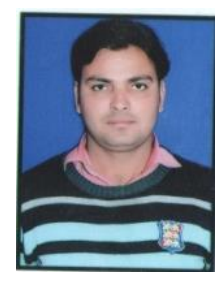

SUDHIR GOSWAMI Student of Master of Technology in CSE at RADHARAMAN Institute of Technology \& Science Bhopal. He has studied his Bachelor of Engineering in CSE at RKDF Institute of Science \& Technology INDIA. His Current Research is in the field of Mobile Adhoc Network. mail Id:10188sudhir@gmail.com

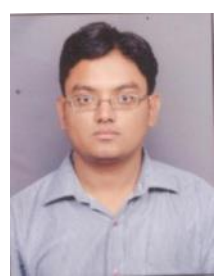

CHETAN AGRAWAL Studied Master of Engineering in CSE at TRUBA Institute of Engineering \& Information Technology INDIA. He has studied his Bachelor of Engineering in CSE at BANSAL Institute of Science \& Technology INDIA. Currently He is working as Assistant professor in CSE department at RADHARAMAN Institute of Technology \& Science Bhopal M.P. INDIA. His research area of interest is Cyber Security, Network Security, Wireless Networks, and Data Mining and Cloud Computing.

Email Id: chetan.agrawal12@gmail.com 